\title{
SOME FUNCTIONALS FOR COPULAS
}

\author{
C. ALSINA \\ Departament de Matemàtiques i Estadistica \\ ETSAB, Universitat Politècnica de Catalunya \\ 08028 Barcelona, Spain. \\ and \\ A. DAMAS and J.J. QUESADA \\ Departamento de Matemática Aplicada \\ Universidad de Granada \\ 18071 Granada, Spain. \\ (Received November 14, 1989 and in revised form April 8, 1990)
}

\begin{abstract}
In this paper we study some functionals operating on the set of the $n$-copulas defined on $[0,1]^{\mathrm{n}}$. Conditions under which such functionals are well defined are determined and some counterexamples are described. The study of the fixed points (n-copulas) for these functionals is also considered, and, finally, some open problems are presented.
\end{abstract}

KEY WORDS AND PHRASES. $N$-increasing functions, connecting copula, $n$-copula. 1980 AMS SUBJECT CLASSIFICATION CODE. 62HO5.

\section{INTRODUCTION.}

In 1959, Abe Sklar [1] introduced the concept of a copula. Since then, theory of copulas has been playing an important role in Mathematics and in particular in Mathematical Statistics and Probability Theory. Many applications of the theory of copulas have been proposed in these parts of Mathematics. In any case, we think that many of the advantages and possibilities of using copulas in Probability Theory remain still to be developed.

The theory of copulas has been found to be of great interest when describing the dependence between two or more random variables, which is given by their joint distribution function.

Several interesting papers dealing with this problem and others have been written in the last few years. We should mention Schweizer and Sklar [2-3], Schweizer and Wolff [4-5], Genest and Mackay [6], and Wolff [7].

In Schweizer and Wolff [4-5] several nonparametric measures of dependence for random variables are defined by using the associated copula. Some properties and interesting results are presented. Wolff [7] has made a detailed study of these measures of dependence.

Genest and Mackay [6] have considered some families of copulas and have studied properties for them. Recently, Alsina and Quesada [8] have studied the relationships between some pairs of copulas.

In this paper, we study some functionals operating on the set of the copulas defined on $[0,1]^{\mathrm{n}}$ and we determine the conditions under which these functionals are well defined. Some counterexamples describing the validity of these conditions are given. We also study the fixed points (n-copulas) for these functionals. We conclude with several open problems. 
In section 2 we give a brief introduction to the theory of copulas. Section 3 is devoted to the study of the functionals defined on the set of copulas. In section 4 we consider the case $\Pi^{*}=\Pi$ and in section 5 we study the copulas that are fixed points for these functionals. Section 6 contains some open problems and conclusions.

2. COPULAS.

A function $\mathrm{C}$ from $[0,1]^{\mathrm{n}}$ into [0.1] is said to be $\mathrm{n}$-increasing if the $\mathrm{C}$-volume of any $\mathrm{n}$-box on $[0,1]^{n}$ is non-negative, that is to say, if $V_{c}(N) \geq 0$ for any $n$-box $N=[(x, y)] \subset[0,1]^{n}$ with $x=\left(x_{1}, \ldots, x_{n}\right), y=\left(y_{1}, \ldots, y_{n}\right), x_{i} \leq y_{i}, i=1,2, \ldots, n$, where the $C$-volume of the $n$-box $N$ is given by

$$
V_{c}(N)=\Sigma \epsilon\left(z_{1}, \ldots, z_{n}\right) \cdot C\left(z_{1}, \ldots, z_{n}\right) \geq 0
$$

where

$$
\epsilon\left(z_{1}, \ldots, z_{n}\right)=\left\{\begin{array}{l}
1 \text { if } z_{i}=x_{i} \text { for an even number of i's. } \\
-1 \text { if } z_{i}=x_{i} \text { for an odd number of i's. }
\end{array}\right.
$$

and the sum in (2.1) is extended to all vertices of $\mathrm{N}$.

Now, let us consider the definition of $n$-copula due to A. Sklar [1] in 1959:

Definition 2.1. A n-dimensional copula or, briefly, a n-copula is a function $C$ from $[0,1]^{\text {n }}$ into $[0,1]$ that satisfies the following conditions:

(i) $C\left(1, \ldots, 1, x_{i}, 1, \ldots, 1\right)=x_{i}$ for every $i$ such that $1 \leq i \leq n$ and every $x_{i} \in[0,1]$.

(ii) $C\left(x_{1}, \ldots, x_{n}\right)=0$ if $x_{i}=0$ for some $i$ with $1 \leq i \leq n$.

(iii) $\mathbf{C}$ is $n$-increasing.

Some easy examples of $n$-copulas are the functions $\Pi, M$ and $W$ respectively defined by

$$
\begin{aligned}
& \Pi\left(x_{1}, x_{2}, \ldots, x_{n}\right)=x_{1} \cdot x_{2} \ldots x_{n} \\
& M\left(x_{1}, x_{2}, \ldots, x_{n}\right)=\operatorname{Min}\left(x_{1}, x_{2}, \ldots, x_{n}\right) \\
& W\left(x_{1}, x_{2}, \ldots, x_{n}\right)=\operatorname{Max}\left(x_{1}+x_{2}+\ldots+x_{n}-n+1,0\right)
\end{aligned}
$$

and while $\Pi$ and $M$ are always $n$-copulas, $W$ is a copula only when $n=2$. It is easy to show that copulas are uniformly continuous functions and that they are increasing in each variable. Also, any copula $C$ satisfies that $W\left(x_{1}, x_{2}, \ldots, x_{n}\right) \leq C\left(x_{1}, x_{2}, \ldots, x_{n}\right) \leq M\left(x_{1}, x_{2}, \ldots, x_{n}\right)$ for every $\left(x_{1}, x_{2}, \ldots, x_{n}\right) \in[0,1]^{n}$.

The fundamental result in the theory of copulas gives us an idea of the importance of a copula, because it is shown that a copula is a function relating the joint distribution function of a random vector with its marginal distribution functions. Namely, the key result in the theory of copulas asserts as follows:

THEOREM 2.2. Let $X_{1}, X_{2}, \ldots, X_{n}$ be $n$ random variables defined on a common probability space and let $F_{1}, F_{2}, \ldots, F_{n}$ and $G$ be the marginal distribution functions and the joint distribution function respectively. Then, there exists a n-copula $C$ such that

$$
G\left(t_{1}, t_{2}, \ldots, t_{n}\right)=C\left(F_{1}\left(t_{1}\right), F_{2}\left(t_{2}\right), \ldots, F_{n}\left(t_{n}\right)\right)
$$

for every $t_{1}, t_{2}, \ldots, t_{n} \in R$. The copula $C$ is called a connecting copula of $X_{1}, X_{2}, \ldots, X_{n}$ and, if $F_{1}, F_{2}, \ldots, F_{n}$ are continuous, then $C$ is unique. Otherwise, $C$ is uniquely determined on $\left(\operatorname{Ran} F_{1}\right) \times\left(\operatorname{Ran} F_{2}\right) \times \ldots \times\left(\operatorname{Ran} F_{n}\right)$.

As a consequence of Theorem 2.2 we can observe that the connecting copula of $\mathrm{n}$ random variables is a function that contains all the information about the dependence of the random variables. This is one the reasons for proposing measures of dependence for random variables in terms of the connecting copula. (See Schweizer and Wolff [4-5] and Wolff [7].

The following result is a consequence of Theorem 2.2. 
THEOREM 2.3. Let $X_{1}, X_{2}, \ldots, X_{n}$ be $n$ random variables defined on a common probability space and having continuons marginal distributions functions. If $\mathrm{C}$ is the connecting copula, then

(i) $\mathrm{C}=\Pi$ if and only if $\mathrm{X}_{1}, \mathrm{X}_{2}, \ldots, \mathrm{X}_{\mathrm{n}}$ are independent.

(ii) $\quad C=M$ if and only if each random variable $X_{i},(i=1,2, \ldots, n)$ is an increasing function of the other ones.

(iii) For $n=2, C=W$ if and only if each random variable $X_{1}(i=1,2)$ is a decreasing function of the other one.

The cases (ii) and (iii) correspond with total and positive, and total and negative dependence of the random variables, respectively.

More results and applications can be found in the abovementioned works and also in the book "Probabilistic Metric Spaces" by Schweizer and Sklar [3], where a whole chapter is devoted to the theory of copulas.

Now, our interest is to study some functionals operating on the set of the $\mathbf{n}$-copulas. These functionals have to be well defined, that is to say, we have to determine the conditions under which the functionals map copulas into copulas.

\section{THE FUNCTIONALS.}

Let $\mathrm{C}$ be any $\mathrm{n}$-copula, and let $\mathrm{P}$ be a fixed $\mathrm{n}$-copula. We consider the functional defined for any $n$-copula $\mathrm{C}$ as follows

$$
\begin{aligned}
C^{*}\left(x_{1}, x_{2}, \ldots, x_{n}\right)= & \lambda . P\left(x_{1}, x_{2}, \ldots, x_{n}\right)+(1-\lambda) .\left[f\left(x_{1}, x_{2}, \ldots, x_{n}\right)\right. \\
& +C\left(\alpha_{1}\left(x_{1}, x_{2}, \ldots, x_{n}\right), \ldots, \alpha_{n}\left(x_{1}, x_{2}, \ldots, x_{n}\right)\right]
\end{aligned}
$$

for every $\left(x_{1}, x_{2}, \ldots, x_{n}\right) \in[0,1]^{n}$, where $f$ is a function defined on $[0,1]^{n}, \alpha_{1}, \alpha_{2}, \ldots, \alpha_{n}$ are functions defined from $[0,1]^{\mathrm{n}}$ into $[0,1]$ and $\lambda \in[0,1]$.

First of all, we want to study conditions for the functions $f, \alpha_{1}, \alpha_{i}, \ldots, \alpha_{n}$ which assure us that $\mathrm{C}^{*}$ is a $\mathrm{n}$-copula for any $\mathrm{n}$-copula. Later, we will consider the case $\Pi^{*}=I$ and we will study the fixed points for these functionals, that is, the n-copulas $\mathrm{C}_{\mathrm{o}}$ such that $\mathrm{C}_{\mathrm{o}}{ }^{*}=\mathrm{C}_{\mathrm{o}}$.

If $\lambda=1$, then $\mathrm{C}^{*}=\mathbf{P}$ for any $\mathrm{n}$-copula $\mathrm{C}$ and it is a trivial case. From now on, we will consider $\lambda \in[0,1)$. In the next result we study conditions on $f, \alpha_{1}, \alpha_{2}, \ldots, \alpha_{n}$ wich ensure that $C^{*}$ satisfies conditions (i) and (ii) of definiton 2.1 for any n-copula $C$.

LEMMA 3.1. Let $\mathrm{C}^{*}$ be as defined in (3.1). For any $n$-copula $\mathrm{C}$ the following statement is true:

(i) $C^{*}\left(1, \ldots, 1, x_{i}, 1, \ldots, 1\right)=x_{i}$ for every $i$ such that $1 \leq i \leq n$ and every $x_{i} \in[0,1]$, and

(ii) $C^{*}\left(x_{1}, \ldots, x_{n}\right)=0$ if $x_{1}=0$ for some $i$ with $1 \leq i \leq n$

if and only if

$$
\begin{aligned}
& f\left(x_{1}, \ldots, x_{n}\right)=x_{1} \ldots x_{n}-C\left(\alpha_{1}\left(x_{1}, \ldots, x_{n}\right), \ldots, \alpha_{n}\left(x_{1}, \ldots, x_{n}\right)\right) \text { for any } \\
& \left(x_{1}, \ldots, x_{n}\right) \in[0,1]^{n} \text { such that } x_{1} \ldots x_{n}=x_{j} \text { for some } j \text { with } 1 \leq j \leq n .
\end{aligned}
$$

PROOF. Suppose $\left(x_{1}, \ldots, x_{n}\right) \in[0,1]^{n}$ such that some $x_{i}=0$ and $C^{*}\left(x_{1}, \ldots, x_{n}\right)=0$. Since $P$ is a copula we have $f\left(x_{1}, \ldots, x_{n}\right)=-C\left(\alpha_{1}\left(x_{1}, \ldots, x_{n}\right), \ldots, \alpha_{n}\left(x_{1}, \ldots, x_{n}\right)\right)$. Similarly, given $\left(1, \ldots, 1, x_{i}, 1, \ldots, 1\right) \in[0,1]^{n}$, if $C^{*}\left(1, \ldots, 1, x_{i}, 1, \ldots, 1\right)=x_{1}$ we have that

$$
\begin{aligned}
x_{i}=\lambda \cdot x_{i} & +(1-\lambda) .\left[f\left(1, \ldots, 1, x_{i}, 1, \ldots, 1\right)+\right. \\
& \left.+C\left(\alpha_{1}\left(1, \ldots, 1, x_{1}, \ldots, 1\right), \ldots, \alpha_{n}\left(1, \ldots, 1, x_{i}, 1, \ldots, 1\right)\right)\right]
\end{aligned}
$$

and since $\lambda \neq 1$ we get the desired expression for $f$. 
In the other direction, let us suppose that

$$
f\left(x_{1}, \ldots, x_{n}\right)=x_{1} \ldots x_{n}-C\left(\alpha_{1}\left(x_{1}, \ldots, x_{n}\right), \ldots, \alpha_{n}\left(x_{1}, \ldots, x_{n}\right)\right)
$$

for any $\left(x_{1}, \ldots, x_{n}\right) \in[0,1]^{n}$ such that $x_{1} \ldots x_{n}=x_{j}$ for some $j, 1 \leq j \leq n$. In this case we get

$$
C^{*}\left(x_{1}, \ldots, x_{n}\right)=\lambda \cdot P\left(x_{1}, \ldots, x_{n}\right)+(1-\lambda) \cdot x_{1} \ldots x_{n}
$$

which completes the proof.

Now, we want to find conditions on $f$ and $\alpha_{1}, \alpha_{2}, \ldots, \alpha_{n}$ independently of the copula $C$, which are necessary and sufficient for $C^{*}$ to satisfy conditions (i) and (ii) of definition 2.1. From now on, we will denote by $I$ the unit interval $[0,1]$, and $I^{n}=[0,1]^{n}$. Also, $J$ will denote the subset of $I^{n}$ given by

$$
J=\left\{\left(x_{1}, \ldots, x_{n}\right) \in I^{n} \mid x_{1} \ldots x_{n}=x_{i}, \text { for some } i, 1 \leq i \leq n\right\}
$$

First, let us prove the following technical result:

PROPOSITION 3.2. If $\Pi^{*}\left(x_{1}, x_{2}, \ldots, x_{n}\right)=M^{*}\left(x_{1}, x_{2}, \ldots, x_{n}\right)$ then for every $\left(x_{1}, x_{2}, \ldots, x_{n}\right) \in J$, there is some $j, 1 \leq j \leq n$, such that $\prod_{i=1}^{n} \alpha_{i}\left(x_{1}, x_{2}, \ldots, x_{n}\right)=\alpha_{j}\left(x_{1}, x_{2}, \ldots, x_{n}\right)$.

PROOF. If $\Pi^{*}\left(x_{1}, x_{2}, \ldots, x_{n}\right)=M^{*}\left(x_{1}, x_{2}, \ldots, x_{n}\right)$ then, by definition,

$$
\Pi\left(\alpha_{1}\left(x_{1}, \ldots, x_{n}\right), \ldots, \alpha_{n}\left(x_{1}, \ldots, x_{n}\right)\right)=M\left(\alpha_{1}\left(x_{1}, \ldots, x_{n}\right), \ldots, \alpha_{n}\left(x_{1}, \ldots, x_{n}\right)\right)
$$

and the result is proved.

LEMMA 3.3. Let $\mathrm{C}^{*}$ be as defined in (3.1). Then the following statement is true:

For every n-copula $\mathbf{C}$,

(i) $C^{*}\left(1, \ldots, 1, x_{i}, 1, \ldots, 1\right)=x_{i}$ for every $i$ such that $1 \leq i \leq n$ and every $x_{i} \in[0,1]$, and

(ii) $C^{*}\left(x_{1}, \ldots, x_{n}\right)=0$ if $x_{i}=0$ for some $i$ with $1 \leq i \leq n$

if and only if

(iii) $f\left(x_{1}, \ldots, x_{n}\right)=x_{1} \ldots x_{n}-\prod_{i=1}^{n} \alpha_{i}\left(x_{1}, \ldots, x_{n}\right)$ for every $\left(x_{1}, \ldots, x_{n}\right) \in J$,

and

(iv) for every $\left(x_{1}, \ldots, x_{n}\right) \in J$ there is some $j, 1 \leq j \leq n$, such that $\prod_{j=1}^{n} \alpha_{i}\left(x_{1}, \ldots, x_{n}\right)=$ $=\alpha_{j}\left(x_{1}, \ldots, x_{n}\right)$.

PROOF. Let $\mathrm{C}^{*}$ be satisfying (i) and (ii), in particular for $\mathrm{C}=\Pi$ and by using lemma 3.1 we get (iii). Also, by hypothesis $\Pi^{*}$ and $\mathrm{M}^{*}$ are in the conditions of proposition 3.2 and (iv) is obtained. In the other direction, let us consider $\left(0, x_{2}, \ldots, x_{n}\right) \in J$, then by (iii).

$$
\begin{aligned}
C^{*}\left(0, x_{2}, \ldots, x_{n}\right)= & (1-\dot{\lambda}) .\left[-\prod_{i=1}^{n} \alpha_{i}\left(0, x_{2}, \ldots, x_{n}\right)+\right. \\
& \left.+C\left(\alpha_{1}\left(0, x_{2}, \ldots, x_{n}\right), \ldots, \alpha_{n}\left(0, x_{2}, \ldots, x_{n}\right)\right)\right]
\end{aligned}
$$

and by (iv) we have $C^{*}\left(0, x_{2}, \ldots, x_{n}\right)=0$. The remaining cases can similarly be proved.

Lemma 3.3 states necessary and sufficient conditions for $f$ and $\alpha_{1}, \alpha_{2}, \ldots, \alpha_{n}$ in order to have $C^{*}$ satisfy (i) and (ii) of the which definition of $n$-copula $C$. It only remains to study conditions guarantee $\mathrm{C}^{*}$ is $\mathrm{n}$-increasing. The theorem provides the solution for this problem.

THEOREM 3.4. Let $\mathrm{C}^{*}$ be as defined in (3.1). If $\mathrm{f}\left(\mathrm{x}_{1}, \ldots, \mathrm{x}_{\mathrm{n}}\right)$ is $\mathrm{n}$-increasing, (iii) and (iv) on proposition 3.3 are satisfied, and either one of the two following conditions hold:

(v) Every function $\alpha_{i}$ depends solely of one variable, which is different for each $\alpha_{1}$. All $\alpha_{i}$ 's are monotone but the number of them that are decreasing, is even.

(vi) There exists one variable $x_{j}(1 \leq j \leq n)$ such that none of the functions $\alpha_{i}$ depends on $x_{j}$ then, 
1) $\mathrm{C}^{*}$ is a $\mathrm{n}$-copula for every $\mathrm{n}$-copula $\mathrm{C}$.

2) If $\mathrm{C}_{1} \leq \mathrm{C}_{2}$, then $\mathrm{C}_{1}{ }^{*} \leq \mathrm{C}_{2}^{*}$.

PROOF. Let us suppose that (v) is satisfied. By applying lemma 3.3, since (iii) and (iv) hold, we have for $\mathrm{C}^{*}$ the conditions (i) and (ii) of definition 2.1. It only remains to prove that $\mathrm{C}^{*}$ is $n$-increasing. Let $N=\left[\left(a_{1}, \ldots, a_{n}\right),\left(b_{1}, \ldots, b_{n}\right)\right]$ be a $n$-box, $N \subset I^{n}$. We have to show that

$$
\Sigma \in\left(z_{1}, \ldots, z_{n}\right) \cdot C^{*}\left(z_{1}, \ldots, z_{n}\right) \geq 0
$$

where the sum is extended to all vertices of $\mathbf{N}$.

Suppose that, by (v), $\alpha_{i}$ only depends on $x_{i}$ for $i=1,2, \ldots, n$. Then, by taking into account that $P$ is a $n$-copula and $f$ is $n$-increasing, (3.2) is equivalent to proving that

$$
\sum \in\left(z_{1}, \ldots, z_{n}\right) . C\left(\left(\alpha_{1}\left(z_{1}\right), \ldots, \alpha_{n}\left(z_{n}\right)\right) \geq 0\right.
$$

Let $c=\left(c_{1}, c_{2}, \ldots, c_{n}\right)$ be given by $c_{i}=\alpha_{i}\left(a_{i}\right)$ if $\alpha_{i}$ is increasing and $c_{i}=\alpha_{i}\left(b_{i}\right)$ if $\alpha_{i}$ is decreasing, and $d=\left(d_{1}, d_{2}, \ldots, d_{n}\right)$ given by $d_{i}=\alpha_{i}\left(b_{i}\right)$ if $\alpha_{i}$ is increasing and $d_{i}=\alpha_{i}\left(a_{1}\right)$ if $\alpha_{i}$ is decreasing. Clearly $N_{1}=[c, d]$ is a $n$-box on $[0,1]^{n}$ and for every vertex $\left(z_{1}, z_{2}, \ldots, z_{n}\right)$ of $N$, we have

$$
\epsilon\left(z_{1}, z_{2}, \ldots, z_{n}\right)=\epsilon\left(\alpha_{1}\left(z_{1}\right), \alpha_{2}\left(z_{2}\right), \ldots, \alpha_{n}\left(z_{n}\right)\right) .
$$

Thus (3.3) is reduced to proving

$$
\sum \in\left(\alpha_{1}\left(\mathrm{z}_{1}\right), \ldots, \alpha_{\mathrm{n}}\left(\mathrm{z}_{\mathrm{n}}\right)\right) . \mathrm{C}\left(\alpha_{1}\left(\mathrm{z}_{1}\right), \ldots, \alpha_{\mathrm{n}}\left(\mathrm{z}_{\mathrm{n}}\right)\right) \geq 0
$$

which is satisfied because $\mathrm{C}$ is a $\mathrm{n}$-copula. Therefore $\mathrm{C}^{*}$ is a $\mathrm{n}$-copula.

Let us suppose that (vi) holds instead of $(v)$. Now, $C^{*}$ will be $n$-increasing if we prove that

$$
\sum \in\left(z_{1}, z_{2}, \ldots, z_{n}\right) . C\left(\alpha_{1}\left(z_{1}, \ldots, z_{n}\right), \ldots, \alpha_{n}\left(z_{1}, \ldots, z_{n}\right)\right) \geq 0
$$

Suppose, for example, that none of the $\alpha_{i}$ 's depends on $x_{1}$, then

$$
\begin{aligned}
\Sigma \in\left(z_{1}, z_{2}, \ldots,\right. & \left.z_{n}\right) . C\left(\alpha_{1}\left(z_{1}, \ldots, z_{n}\right), \ldots, \alpha_{n}\left(z_{1}, \ldots, z_{n}\right)\right)= \\
& =\Sigma \in\left(a_{1}, z_{2}, \ldots, z_{n}\right) . C\left(\alpha_{1}\left(a_{1}, \ldots, z_{n}\right), \ldots, \alpha_{n}\left(a_{1}, \ldots, z_{n}\right)\right)+ \\
& +\Sigma \in\left(b_{1}, z_{2}, \ldots, z_{n}\right) . C\left(\alpha_{1}\left(b_{1}, \ldots, z_{n}\right), \ldots, \alpha_{n}\left(b_{1}, \ldots, z_{n}\right)\right)=0
\end{aligned}
$$

because $\epsilon\left(a_{1}, z_{2}, \ldots, z_{n}\right)=-\epsilon\left(b_{1}, z_{2}, \ldots, z_{n}\right)$ and $\alpha_{i}\left(a_{1}, z_{2}, \ldots, z_{n}\right)=\alpha_{i}\left(b_{1}, z_{2}, \ldots, z_{n}\right)$ for $\mathrm{i}=1,2, \ldots, \mathrm{n}$.

It is obvious that if $\mathrm{C}_{1} \leq \mathrm{C}_{2}$, then $\mathrm{C}_{1}^{*} \leq \mathrm{C}_{2}^{*}$.

COUNTEREXAMPLE. It seems reasonable to think that conditions ( $v$ ) and (vi) in theorem 3.4 could be too strong. This is not the case, and to illustrate this, we give the following counterexample which shows us that either one of those hypothesis are needed. Let $C^{*}(x, y)$ be given by

$$
C^{*}(x, y)=\lambda \cdot \Pi(x, y)+(1-\lambda) \cdot\left[f(x, y)+C\left(\alpha_{1}(x, y), \alpha_{2}(x, y)\right)\right]
$$

and let us consider $\alpha_{1}(x, y)=\operatorname{Min}\{y \cdot(x+y), y\}, \alpha_{2}(x, y)=x$ and $f(x, y)=0$. Then $f$ is 2-increasing and (iii) and (iv) are satisfied, but neither (v) or (vi) hold. Also, we observe that $\alpha_{1}$ and $\alpha_{2}$ are increasing and continuous functions and therefore we eliminate the idea that continuity could change the conditions of our problem. If we consider the values $x_{1}=3 / 4, x_{2}=1, y_{1}=1 / 8$, $y_{2}=1 / 2$ and $C=M$, then we get

$$
\begin{aligned}
M^{*}(3 / 4,1 / 8) & +M^{*}(1,1 / 2)-M^{*}(3 / 4,1 / 2)-M^{*}(1,1 / 8)= \\
& =\lambda .(1 / 2-1 / 8) .(1-3 / 4)+(1-\lambda) .(-1 / 64)
\end{aligned}
$$

which is negative for some values of $\lambda$, and therefore $M^{*}$ is not 2-increasing. 


\section{THE CASE $\Pi^{*}=\Pi$.}

Once we know the conditions for $\mathrm{C}^{*}$ being a $\mathrm{n}$-copula, we are interested in the study of the particular cases in which the $n$-copula $\Pi$ remains fixed, that is to say $\Pi^{*}=\Pi$. The theorem provides the solution for this problem:

THEOREM 4.1. Let $\mathrm{C}^{*}$ be as defined in (3.1). The following conditions are equivalent:

(i) $\Pi^{*}=\Pi$.

(ii) $f\left(x_{1}, \ldots, x_{n}\right)=\frac{x_{1} \ldots x_{n}-\lambda . P\left(x_{1}, \ldots, x_{n}\right)}{1-\lambda}$

for every $\left(x_{1}, \ldots, x_{n}\right) \in I^{n}$.

(iii) $C^{*}\left(x_{1}, \ldots, x_{n}\right)=x_{1} \ldots x_{n}+$

$$
+(1-\lambda) \cdot\left[C\left(\alpha_{1}\left(x_{1}, \ldots, x_{n}\right), \ldots, \alpha_{n}\left(x_{1}, \ldots, x_{n}\right)\right)-\prod_{i=1}^{n} \alpha_{i}\left(x_{1}, \ldots, x_{n}\right)\right]
$$

for every $\left(x_{1}, \ldots, x_{n}\right) \in I^{n}$.

PROOF. (i) $\Rightarrow$ (ii). If $\Pi^{*}=\Pi$, then

$$
\lambda . P\left(x_{1}, \ldots, x_{n}\right)+(1-\lambda) .\left[f\left(x_{1}, \ldots, x_{n}\right)+\prod_{i=1}^{n}\left(\alpha_{i}\left(x_{1}, \ldots, x_{n}\right)\right]=x_{1} \ldots x_{n}\right.
$$

and we get (ii).

(ii) $\Rightarrow$ (iii). By substituting $f$ in the general expression of $C^{*}$ we have (iii).

(iii) $\Rightarrow$ (i). If we put $\mathrm{C}=\Pi$ in (iii), we get $\Pi^{*}=\Pi$.

We observe that $C^{*}$ in (iii) can be seen in the general form (3.1) where $P=\Pi$ and $f$ is given by $f\left(x_{1}, \ldots, x_{n}\right)=x_{1} \ldots x_{n}-\prod_{i=1}^{n} \alpha_{i}\left(x_{1}, \ldots, x_{n}\right)$ for every $\left(x_{1}, \ldots, x_{n}\right) \in I^{n}$. We will use this fact to prove the following result:

THEOREM 4.2. Let $C^{*}$ be given by (iii) in theorem 4.1. If there exists one variable $x_{j}$ $(1 \leq j \leq n)$ such that none of the functions $\alpha_{i}$ depends on $x_{j}$, and for every $\left(x_{1}, \ldots, x_{n}\right) \in J$ there is some $k, 1 \leq \mathrm{k} \leq \mathrm{n}$, such that $\prod_{\mathrm{i}=1}^{\mathrm{n}} \alpha_{\mathrm{i}}\left(\mathrm{x}_{1}, \ldots, \mathrm{x}_{\mathrm{n}}\right)=\alpha_{\mathrm{k}}\left(\mathrm{x}_{1}, \ldots, \mathrm{x}_{\mathrm{n}}\right)$, then

1) $C^{*}$ is a $n$-copula for every $n$-copula $C$.

2) $\Pi^{*}=\Pi$.

3) For every n-copula $C, C \geq \Pi \Rightarrow C^{*} \geq \Pi$ and $C \leq \Pi \Rightarrow C^{*} \leq \Pi$.

PROOF. Since $C^{*}$ has the general form (3.1) where $P=\Pi$ and

$$
f\left(x_{1}, \ldots, x_{n}\right)=x_{1} \ldots x_{n}-\prod_{i=1}^{n} \alpha_{i}\left(x_{1}, \ldots, x_{n}\right),
$$

and by using the second hypothesis of the functions $\alpha_{i}$, we can apply lemma 3.3 and therefore $C^{*}$ satisfies conditions (i) and (ii) of definition 2.1. In order to be able to use theorem 3.4 which assures us that $C^{*}$ is a $n$-copula, it is enough to show that $f\left(x_{1}, \ldots, x_{n}\right)$ is $n$-increasing.

Let $N=\left[\left(a_{1}, \ldots, a_{n}\right),\left(b_{1}, \ldots, b_{n}\right)\right]$ be a $n$-box in $I^{n}$, and suppose, for example, that none of the funtions $\alpha_{i}$ depends on $x_{1}$. Then,

$$
\begin{aligned}
\Sigma \in\left(\mathrm{z}_{1}, \mathrm{z}_{2}, \ldots, \mathrm{z}_{\mathrm{n}}\right) \cdot \prod_{\mathrm{i}=1}^{\mathrm{n}} & \alpha_{\mathrm{i}}\left(\mathrm{z}_{1}, \mathrm{z}_{2}, \ldots, \mathrm{z}_{\mathrm{n}}\right)= \\
= & \Sigma \in\left(\mathrm{a}_{1}, \mathrm{z}_{2}, \ldots, \mathrm{z}_{\mathrm{n}}\right) \cdot \prod_{\mathrm{i}=1}^{n} \alpha_{i}\left(\mathrm{a}_{1}, \mathrm{z}_{2}, \ldots, \mathrm{z}_{\mathrm{n}}\right) \\
& +\Sigma \in\left(\mathrm{b}_{1}, \mathrm{z}_{2}, \ldots, \mathrm{z}_{\mathrm{n}}\right) \cdot \prod_{\mathrm{i}=1}^{\mathrm{n}} \alpha_{\mathrm{i}}\left(\mathrm{b}_{1}, \mathrm{z}_{2}, \ldots, \mathrm{z}_{\mathrm{n}}\right)=0
\end{aligned}
$$

because $\epsilon\left(a_{1}, z_{2}, \ldots, z_{n}\right)=-\epsilon\left(b_{1}, z_{2}, \ldots, z_{n}\right)$ and $\alpha_{i}\left(a_{1}, z_{2}, \ldots, z_{n}\right)=\alpha_{i}\left(b_{1}, z_{2}, \ldots, z_{n}\right)$ for every 
$\mathrm{i}, 1 \leq \mathrm{i} \leq \mathrm{n}$. Therefore,

$$
\sum \in\left(z_{1}, \ldots, z_{n}\right) . f\left(z_{1}, \ldots, z_{n}\right)=\sum \in\left(z_{1}, \ldots, z_{n}\right) . z_{1} \ldots z_{n} \geq 0
$$

and $C^{*}$ is a $n$-copula. By theorem 4.1 , it is clear that $\Pi^{*}=\Pi$, and the proof of 3 ) is obvious.

REMARK. We can observe that hypothesis (v) of theorem 3.4 does not work in theorem 4.2. Let us suppose, for example, that $\alpha_{i}$ only depends on $x_{1}$ for $i=1,2, \ldots, n$, and the number of functions $\alpha_{\mathrm{i}}$ that are decreasing is even. In this case we have that

$$
\Sigma \in\left(z_{1}, \ldots, z_{n}\right) . C\left(\alpha_{1}\left(z_{1}\right), \ldots, \alpha_{n}\left(z_{n}\right)\right) \geq 0,
$$

and in order to assure that $\mathrm{C}^{*}$ is $\mathrm{n}$-increasing we have to prove that $\mathrm{f}$ is $\mathrm{n}$-increasing, that is to say,

$$
-\Sigma \in\left(z_{1}, z_{2}, \ldots, z_{n}\right) \cdot \alpha_{1}\left(z_{1}\right) \cdot \alpha_{2}\left(z_{2}\right) \ldots \alpha_{n}\left(z_{n}\right) \geq 0 .
$$

But,

$$
\begin{aligned}
&-\Sigma \in\left(z_{1}, z_{2}, \ldots, z_{n}\right) \cdot \alpha_{1}\left(z_{1}\right) \cdot \alpha_{2}\left(z_{2}\right) \ldots \alpha_{n}\left(z_{n}\right)= \\
&-\Sigma \in\left(b_{1}, z_{2}, \ldots, z_{n}\right) \cdot \alpha_{1}\left(b_{1}\right) \cdot \alpha_{2}\left(z_{2}\right) \ldots \alpha_{n}\left(z_{n}\right)+ \\
&+\Sigma \in\left(b_{1}, z_{2}, \ldots, z_{n}\right) \cdot \alpha_{1}\left(a_{1}\right) \cdot \alpha_{2}\left(z_{2}\right) \ldots \alpha_{n}\left(z_{n}\right)= \\
&=-\Sigma \in\left(b_{1}, z_{2}, \ldots, z_{n}\right) \cdot\left[\alpha_{1}\left(b_{1}\right)-\alpha_{1}\left(a_{1}\right)\right] \cdot \alpha_{2}\left(z_{2}\right) \ldots \alpha_{n}\left(z_{n}\right) .
\end{aligned}
$$

Repeating the same process we can obtain

$$
-\Sigma \in\left(z_{1}, z_{2}, \ldots, z_{n}\right) \cdot \alpha_{1}\left(z_{1}\right) \cdot \alpha_{2}\left(z_{2}\right) \ldots \alpha_{n}\left(z_{n}\right)=-\prod_{i=1}^{n}\left[\alpha_{i}\left(b_{i}\right)-\alpha_{i}\left(a_{i}\right)\right] \leq 0
$$

because, by $(v)$ all the functions $\alpha_{i}$ are monotone and the number of them that are decreasing is even, and therefore $f\left(x_{1}, x_{2}, \ldots, x_{n}\right)$ is not $n$-increasing.

\section{FIXED COPULAS.}

Now, we are interested in finding these copulas that remain fixed for the functionals we are studying. First, we consider $\lambda \in(0,1)$ and then we will see in next theorem that the answer for this problem is the existence and uniqueness of a n-copula $C_{0}$ such that $C_{o}{ }^{*}=C_{0}$.

THEOREM 5.1. Let $C^{*}$ be as defined in (3.1), and $\lambda \in(0,1)$.

If $f\left(x_{1}, \ldots, x_{n}\right)=P\left(x_{1}, \ldots, x_{n}\right)-P\left(\alpha_{1}\left(x_{1}, \ldots, x_{n}\right), \ldots, \alpha_{n}\left(x_{1}, \ldots, x_{n}\right)\right)$, then $\mathrm{C}^{*}=\mathrm{C} \Leftrightarrow \mathrm{C}=\mathrm{P}$.

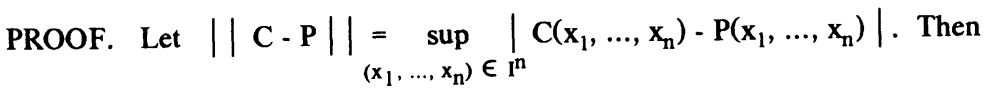

$$
\begin{aligned}
|| C^{*}-P|| & =|1-\lambda| \sup _{\left(x_{1}, \ldots, x_{n}\right) \in I^{n}}\left|C\left(\alpha_{1}, \ldots, \alpha_{n}\right)-P\left(\alpha_{1}, \ldots, \alpha_{n}\right)\right| \leq \\
& \leq|1-\lambda| \cdot \sup _{\left(x_{1}, \ldots, x_{n}\right) \in I^{n}} C\left(x_{1}, \ldots, x_{n}\right)-P\left(x_{1}, \ldots, x_{n}\right) \mid= \\
& =|1-\lambda| \cdot|| C-P||,
\end{aligned}
$$

which concludes the proof.

COROLLARY 5.2. Let $\mathrm{C}^{*}$ be as in theorem 5.1. Then, the following is true:

(i) $\mathrm{C}^{* *}=\mathrm{C} \Leftrightarrow \mathrm{C}=\mathrm{P}$

(ii) If $C_{o}=C$ and $C_{n+1}=C_{n}^{*}$, for $n=0,1,2, \ldots$, then $\left\{C_{n}\right\} \quad \cdots . . P$.

PROOF. From theorem 5.1. (i) is unisspelled. For proving (ii) it is enough to see that $\left\|C_{n}-P\right\| \leq|1-\lambda| n \cdot\|C-P\|$ and therefore when $n$ increases the $n$-copula $C_{n}$ is as close as we wish to the $n$-copula $P$.

The result gives us the relationships between the unique n-copula that is fixed for a functional, and the functional itself. 
COROLLARY 5.3. Let $C^{*}$ be as defined in (3.1) with $\lambda=\lambda_{o} \in(0,1)$, and let $C_{o}$ be any n-copula. The following conditions are equivalent:

(i) $\mathrm{C}_{\mathrm{o}}^{*}=\mathrm{C}_{\mathrm{o}}$.

(ii) $C^{*}\left(x_{1}, \ldots, x_{n}\right)=C_{0}\left(x_{1}, \ldots, x_{n}\right)+\left(1-\lambda_{0}\right) .\left[C\left(\alpha_{1}, \ldots, \alpha_{n}\right)-C_{o}\left(\alpha_{1}, \ldots, \alpha_{n}\right)\right]$.

(iii) $\mathrm{C}_{\mathrm{o}}{ }^{*}=\mathrm{C}_{\mathrm{o}}$ and $\mathrm{C}_{\mathrm{o}}$ is the only $\mathrm{n}$-copula that is fixed for this functional.

PROOF. (i) $\Rightarrow$ (ii). If $\mathrm{C}_{\mathrm{o}}{ }^{*}=\mathrm{C}_{\mathrm{o}}$, then

$$
\begin{aligned}
C_{o}\left(x_{1}, \ldots, x_{n}\right)=\lambda_{0} \cdot P\left(x_{1}, \ldots, x_{n}\right) & +\left(1-\lambda_{0}\right) \cdot\left[f\left(x_{1}, \ldots, x_{n}\right)+\right. \\
& \left.+C_{o}\left(\alpha_{1}\left(x_{1}, \ldots, x_{n}\right), \ldots, \alpha_{n}\left(x_{1}, \ldots, x_{n}\right)\right)\right]
\end{aligned}
$$

Therefore,

$$
f\left(x_{1}, \ldots, x_{n}\right)=\frac{C_{0}\left(x_{1}, \ldots, x_{n}\right)-\lambda_{0} \cdot P\left(x_{1}, \ldots, x_{n}\right)}{1-\lambda_{0}}-C_{o}\left(\alpha_{1}, \ldots, \alpha_{n}\right)
$$

and by substituting in the expression of $\mathrm{C}^{*}$ we obtain (ii).

(ii) $\Rightarrow$ (iii). We can write (ii) in the following way

$$
\begin{aligned}
C^{*}\left(x_{1}, \ldots, x_{n}\right)=\lambda_{0} \cdot C_{0}\left(x_{1}, \ldots, x_{n}\right)+ & \left(1-\lambda_{0}\right) .\left[C\left(\alpha_{1}, \ldots, \alpha_{n}\right)+\right. \\
& \left.+C_{0}\left(x_{1}, \ldots, x_{n}\right)-C_{0}\left(\alpha_{1}, \ldots, \alpha_{n}\right)\right]
\end{aligned}
$$

By applying theorem 5.1, where $\mathrm{P}\left(\mathrm{x}_{1}, \ldots, \mathrm{x}_{\mathrm{n}}\right)=\mathrm{C}_{\mathrm{o}}\left(\mathrm{x}_{1}, \ldots, \mathrm{x}_{\mathrm{n}}\right)$ and

$$
f\left(x_{1}, \ldots, x_{n}\right)=C_{0}\left(x_{1}, \ldots, x_{n}\right)-C_{o}\left(\alpha_{1}\left(x_{1}, \ldots, x_{n}\right), \ldots, \alpha_{n}\left(x_{1}, \ldots, x_{n}\right)\right),
$$

we obtain (iii). On the other hand (iii) $\Rightarrow$ (i) is trivial.

Now we will study conditions under which the functional will have the property that $\mathrm{C}^{* *}=\mathrm{C}$ for every $\mathrm{n}$-copula $\mathrm{C}$. The result gives us conditions for $\alpha_{1}, \alpha_{2}, \ldots, \alpha_{\mathrm{n}}$ in particular cases of $\mathrm{C}^{*}$ where that property holds.

PROPOSITION 5.4. Let $\mathrm{C}^{*}$ be given by

$$
C^{*}\left(x_{1}, \ldots, x_{n}\right)=\prod_{i=1}^{n} x_{i}-\prod_{i=1}^{n} \alpha_{i}\left(x_{1}, \ldots, x_{n}\right)+C\left(\alpha_{1}, \ldots, \alpha_{n}\right) .
$$

If $\alpha_{i}\left(\alpha_{1}\left(x_{1}, \ldots, x_{n}\right), \ldots, \alpha_{n}\left(x_{1}, \ldots, x_{n}\right)\right)=x_{1}$ for $i=1,2, \ldots, n$, then $C^{* *}=C$ for every n-copula $C$.

PROOF. This case corresponds with $\lambda=0$ and $\Pi^{*}=\Pi$. If we compute $C^{* *}$ we obtain

$$
C^{* *}\left(x_{1}, \ldots, x_{n}\right)=\prod_{i=1}^{n} x_{i}-\prod_{i=1}^{n} \alpha_{i}\left(x_{1}, \ldots, x_{n}\right)+C^{*}\left(\alpha_{1}, \ldots, \alpha_{n}\right)=C\left(x_{1}, \ldots, x_{n}\right) .
$$

\section{CONCLUSIONS AND REMARKS.}

The results presented in this paper are interesting in their own right, and lead us to several open problems. A brief descriptions of some of them follows.

First, let $F_{1}, F_{2}, \ldots, F_{n}$ be the marginal distribution functions of a random vector and let $F$ be its joint distribution function and let us suppose that $C$ is a connecting copula associated with $F$. If $\mathrm{C}^{*}$ is the n-copula that we obtain when applying the functional defined by $(3.1)$ to $C$, then the problem is to find the relationships between $F$ and $F^{*}$, where $F^{*}$ is the joint distribution function of a random vector having $F_{1}, F_{2}, \ldots, F_{n}$ as marginal distribution functions and $C^{*}$ as a connecting copula. In short, the problem consists in finding the relationships between the joint distribution functions with connecting $\mathrm{n}$-copulas $\mathrm{C}$ and $\mathrm{C}^{*}$.

Another problem is to generalize the functional in the sense that the one defined here could be seen as a particular case. We observe that if we consider

$$
C\left(x_{1}, \ldots, x_{n}\right)=\lambda \cdot P\left(x_{1}, \ldots, x_{n}\right)+(1-\lambda) .\left[f\left(x_{1}, \ldots, x_{n}\right)-C\left(\alpha_{1}, \ldots, \alpha_{n}\right)\right],
$$


then similar properties and results for this functional can be shown. In any case the problem is to extend these two cases to a more general functional.

It would also be very interesting to study the copulas that we obtain when applying the functional to some known copulas.

ACKNOWLEDGEMENT. The authors would like to thank the referee for helpful comments which improved the presentation of the paper.

\section{REFERENCES.}

1. Sklar, A. "Functions de répartion à n dimensions et leurs marges". Publ. Inst. Statisf. Univ. Paris 8, (1959), 229 - 231.

2. Schweizer, B. and Sklar, A. "Mesure aleátoire de l'information et mésure de l'information par un ensemble d'observateurs". C.R. Acad. Sci. Paris 272A. (1971), 149 - 152.

3. Schweizer, B. and Sklar, A. "Probabilistic Metric Spaces". Elsevier Science Publishing, Co., Inc., New York, (1983).

4. Schweizer, B. and Wolff, E.F. "Sur une mesure de dépendence pour les variables aléatoires". C.R. Acad. Sci. Paris 283A, (1976), 659 - 661.

5. Schweizer, B. and Wolff, E.F. "On nonparametric measures of dependence for random variables". Ann. Statist. 9, (1981), 879 - 885.

6. Genest, C. and Mackay, R.J. "Copulas archimédiennes et familles de lois bidimensionelles dont les marges sont données". Revue Canadienne de Statistique v. 14 N2, (1986), $145 \cdot 159$.

7. Wolff, E.F. "Measures of dependence derived from copulas". Ph. D. Thesis, Univ. of Massachusetts, (1977).

8. Alsina, C. and Quesada, J.J. "On the associativity of C (x,y) and $x-C(x, 1-y)$ ". Proc. 18th. Int. Symp. on Multiple-Valued Logic, Palma de Mallorca, Spain, (1988). 52 - 53.

9. Sklar, A. "Random variables, joint distribution functions, and copulas". Kybernetika 9, (1973), 449 - 460. 


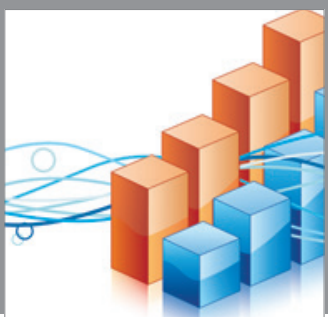

Advances in

Operations Research

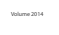

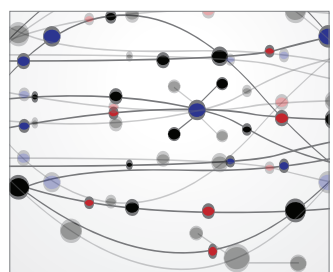

\section{The Scientific} World Journal
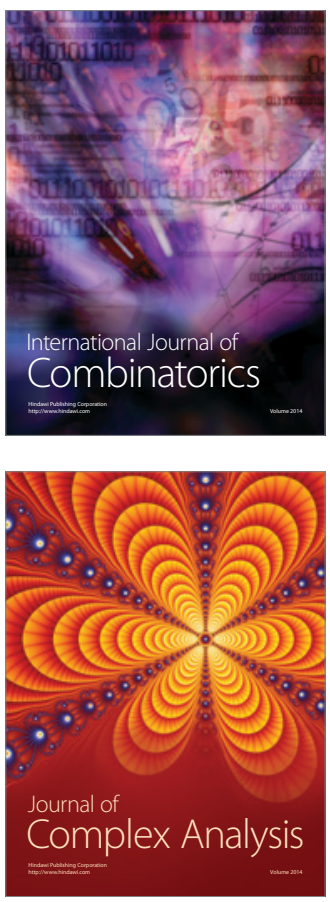

International Journal of

Mathematics and

Mathematical

Sciences
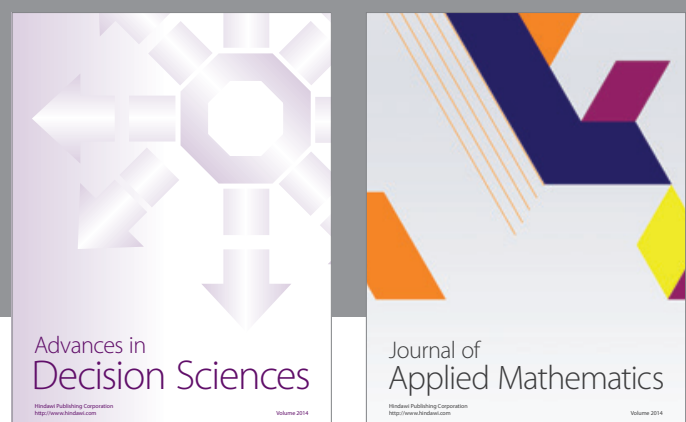

Journal of

Applied Mathematics
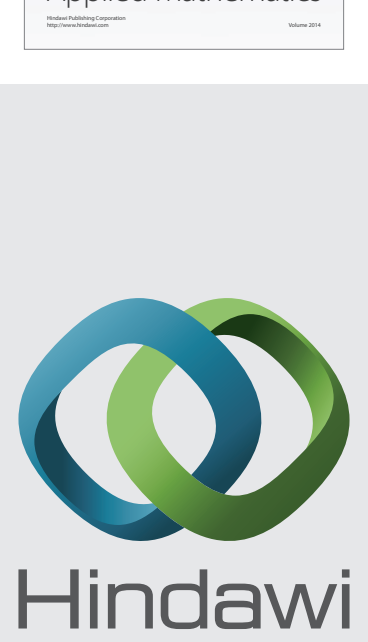

Submit your manuscripts at http://www.hindawi.com
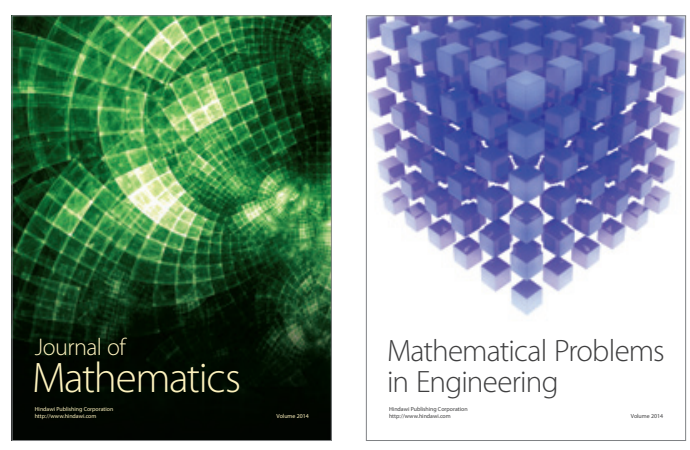

Mathematical Problems in Engineering
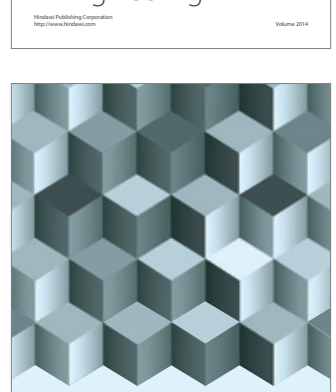

Journal of

Function Spaces
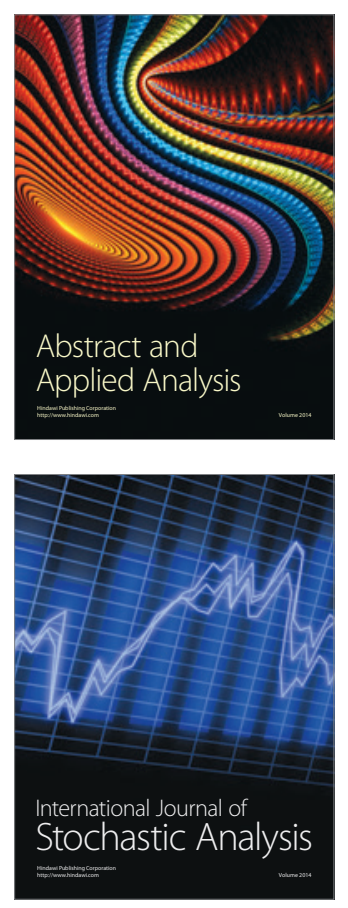

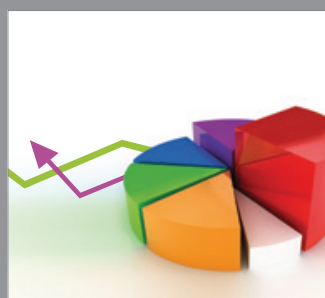

ournal of

Probability and Statistics

Promensencen
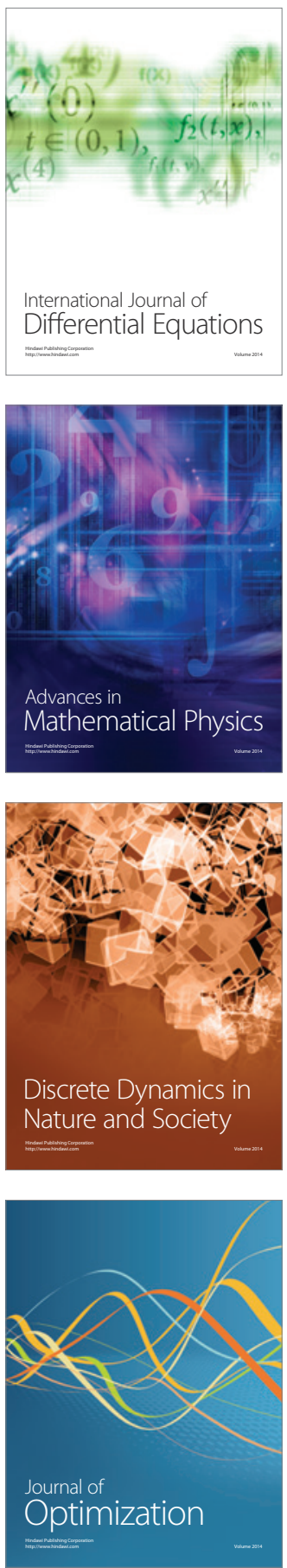